\title{
Survival analysis of second primary malignancies after cervical cancer using a competing risk model: implications for prevention and surveillance
}

\author{
Runmei Li^, Yue Zhang, Bingqing $\mathrm{Ma}^{\wedge}$, Kangming Tan^, Henry S. Lynn^, Zhenyu Wu^ \\ Department of Biostatistics, School of Public Health, Fudan University, Shanghai, China \\ Contributions: (I) Conception and design: R Li, HS Lynn, Z Wu; (II) Administrative support: HS Lynn, Z Wu; (III) Provision of study materials \\ or patients: R Li, B Ma, K Tan, Y Zhang; (IV) Collection and assembly of data: Z Wu; (V) Data analysis and interpretation: R Li, B Ma, K Tan, Y \\ Zhang; (VI) Manuscript writing: All authors; (VII) Final approval of manuscript: All authors. \\ Correspondence to: Prof. Henry S. Lynn; Dr. Zhenyu Wu. Department of Biostatistics, School of Public Health, Fudan University, No. 130 Dongan \\ Rd, Xuhui District, Shanghai 200032, China. Email: hslynn@shmu.edu.cn; zyw@fudan.edu.cn.
}

\begin{abstract}
Background: Previous studies have reported an increased risk for second primary malignancies (SPMs) after cervical cancer (CC). This study aims to quantify and assess the risk of developing SPMs in long-term survivors of CC.

Methods: A population-based cohort of CC patients aged 20-79 years was obtained from the Surveillance, Epidemiology, and End Results (SEER) database. A competing risk model and corresponding nomogram were constructed to predict the 3-, 5-, and 10-year cumulative risks of SPMs. A Fine-Gray plot was created to validate the model. Finally, we performed decision curve analysis (DCA) to evaluate the clinical usefulness of the model by calculating the net benefit.

Results: A total of 34,295 patients were identified, and approximately $6.3 \%$ of the study participants developed SPMs. According to the multivariable competing-risk model, older black CC survivors with localized disease who were treated with radiation therapy were more susceptible to SPMs. The 3-, 5-, and 10-year cumulative incidences of SPMs were 2.5\%, 3.6\%, and 6.2\%, respectively. Calibration curves showed good agreement between the predicted and observed models. The DCA yielded a wide range of risk thresholds at which the net benefits could be obtained from our proposed model.

Conclusions: This study provides physicians with a practical, individualized prognostic estimate to assess the risk of SPMs among CC survivors. CC survivors remain at a high risk of developing SPMs, and further surveillance should focus especially on the patients with black race, older age, localized disease, or those having received radiation therapy.
\end{abstract}

Keywords: Cervical cancer (CC); nomogram; surveillance; second primary malignancies (SPMs); competing risk model

Submitted Feb 27, 2020. Accepted for publication Oct 23, 2020.

doi: $10.21037 /$ atm-20-2003

View this article at: http://dx.doi.org/10.21037/atm-20-2003

\footnotetext{
^ ORCID: Runmei Li, 0000-0002-8739-2929; Bingqing Ma, 0000-0001-7556-4650; Kangming Tan, 0000-0001-9617-9372; Henry S. Lynn, 0000-0002-8078-5690; Zhenyu Wu, 0000-0002-0133-2117.
} 


\section{Introduction}

Cervical cancer (CC) represents $6.6 \%$ of all female cancers and is the $4^{\text {th }}$ leading cancer in women worldwide (1), accounting for approximately 569,000 new cases and 311,000 new deaths annually (2). Depending on the clinical stage and histological type, the 5-year survival rate of CC patients differs slightly. Generally, it reaches $60-85 \%$ in stage I, $30-60 \%$ in stage II, $20-40 \%$ in stage III, and less than $15 \%$ in stage IV. Adenocarcinomas of the cervix have a worse prognosis than squamous cell cancers (3). For patients with early-stage CC, surgery and adjuvant chemotherapy or radiotherapy continue to be the main treatment approaches. In contrast, for patients with advanced-stage (stage IVB), systemic chemotherapy remains the first choice (4). Increased screening has resulted in the earlier detection of CC and the extensive application of advanced therapeutic techniques in clinical practice, and the survival of CC patients has therefore improved significantly (5). However, most CC survivors still face several long-term risks, including recurrence, metastasis, or the development of second primary malignancies (SPMs) $(5,6)$. SPMs are histologically different from primary cancer and occur at a different site (7). Moreover, SPMs now account for approximately $17 \%$ of all incident cancers reported each year to the National Cancer Institute's Surveillance, Epidemiology, and End Results (SEER) program (8). Population-based studies pointed out that CC survivors were at great risk of developing SPMs (9), and these patients were even more likely to die from their SPMs than from their initial cancers (10).

Several studies showed that age (11) and therapies (12) were associated with the development of SPMs in patients with CC. However, previous studies mostly focused on the overall risk relative to the general population and failed to provide the individualized risk of SPMs. In addition, the traditional survival analyses such as standard Kaplan-Meier (KM) and Cox regression methods used in these studies do not consider death as a competing event to SPMs, thus leading to an overestimated risk of developing SPMs (13).

In this study, we aim to comprehensively compare the characteristics of CC patients with and without SPMs using a large population-based cohort, and to establish a competing-risk nomogram to identify the potential risk factors of SPMs after the diagnosis of CC. The corresponding nomogram was also validated for clinical convenience. We present this article in accordance with the
TRIPOD reporting checklist (14) (available at http://dx.doi. org/10.21037/atm-20-2003).

\section{Methods}

\section{Patients}

Data were extracted from the November 2017 release [1992-2015] of the Surveillance, Epidemiology, and End Results (SEER) database. The SEER database collected information on demographics, and cancer incidences, characteristics, and survival from cancer registries across the United States (15). CC patients were identified according to the International Classification of Diseases of Oncology, 3rd edition (ICD-O-3) site code (C53.0, Endocervix; C53.1, Exocervix; C53.8, Overlapping lesion of cervix uteri; C53.9, Cervix uteri).

The study period was restricted from 1998 to 2010 because 1998 was the first year that complete surgery information was available. We limited the analyses to patients diagnosed before 2010 to ensure at least a 5 -year follow-up after the initial diagnosis. As suggested in the SEER database, we considered patients histologically diagnosed with a new malignancy 2 months or more after the diagnosis of the first primary cancer as SPM patients (16). Furthermore, patients were considered qualified if (I) their diagnosis was not reported from death certificate or autopsy; (II) they were aged between 20 and 79; (III) essential covariates were not missing. We subsequently excluded CC patients with SPMs diagnosed within 2 months after the initial diagnosis (Figure 1).

\section{Study outcome and covariates}

The outcome of interest in this study was the occurrence of an SPM. Underlying covariates included patients' demographics (age and race), clinicopathological characteristics (tumor grade, SEER stage, and histologic type), and treatments to the initial CC (radiotherapy and surgery).

\section{Statistical analyses}

Statistical analyses and plotting were performed with $\mathrm{R}$ (version 3.5.3; R Foundation for Statistical Computing, Vienna, Austria). The overview of the study design is shown in Figure S1. The characteristics of patients from the SEER cohort were summarized with counts and 


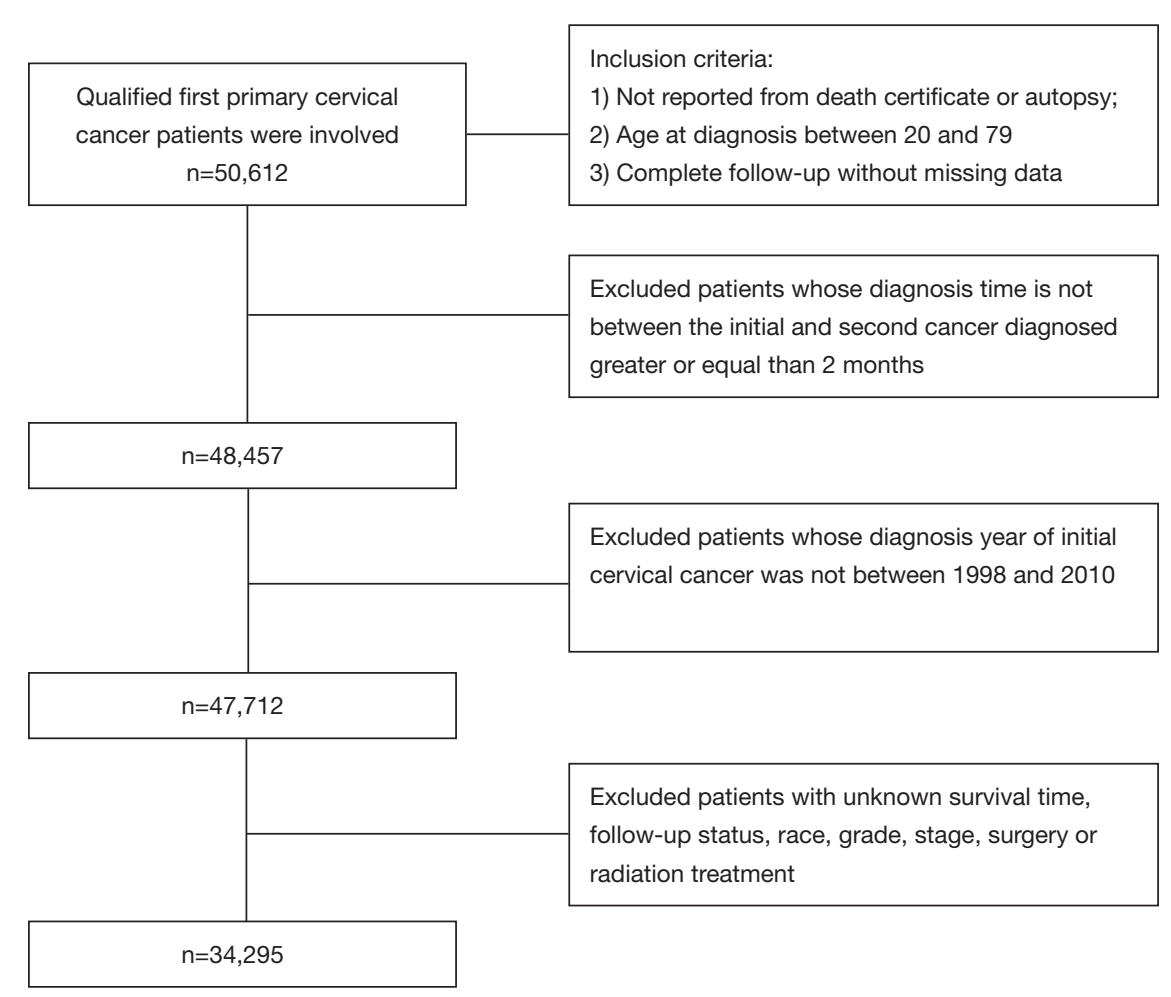

Figure 1 Flow diagram of patients enrolled from the Surveillance, Epidemiology, and End Results (SEER) research database.

percentages, and were compared by the Pearson chi-square test. In this sample, a considerable proportion of patients died before the development of SPMs, and a conventional Cox proportional hazard regression analysis, which cannot consider death as a competing event, would lead to overestimated hazard risks (17). Therefore, the Fine and Gray subdistribution hazard model was used to estimate the risk of the occurrence of SPMs in the presence of competing risk (13) using the $\mathrm{R}$ package cmprsk. To select the most relevant potential factors in the final prediction model, a backward stepwise method was applied based on the Bayesian Information Criterion (BIC) (18) using the package crrstep in R. Cumulative incidence function (CIF) plots were generated to show the estimated probabilities of developing SPMs over time. KM plots were used to describe the overall survival (OS) of enrolled patients. When the KM curves for those with and without SPMs crossed with each other, the difference in restricted mean survival times (RMST) between the two groups was tested (19) using package survRM2 in R.

To quantitatively generate individualized predictions and identify high-risk patients (20), a competing-risk nomogram was established to estimate the 3-, 5-, and 10-year probabilities of developing an SPM by incorporating the points of significant predictors based on the final model using the $\mathrm{R}$ packages nomogramEx and nomogramFormula. Calibration was assessed by plotting the observed probabilities against the expected probabilities via a bootstrap resampling method with 200 bootstrap samples. Additionally, decision curve analysis (DCA), a novel method to provide a direct clinical interpretation, was applied to calculate the net benefit using the rates of true and false positives for various screening thresholds (21).

A two-sided $\mathrm{P}$ value less than 0.05 was considered statistically significant, and the results of all survival models were presented as subdistribution hazard ratios (sdHR) with corresponding $95 \%$ confidence intervals (95\% CI).

\section{Results}

\section{Characteristics of the study population}

A total of 34,295 patients were included in the present study, among whom 2,154 (6.3\%) developed SPMs during the 18 -year follow-up. The mean age at initial 
Table 1 Demographic and clinicopathological characteristics of cervical cancers patients with and without SPMs

\begin{tabular}{|c|c|c|c|c|}
\hline Variable/characteristics & Overall, N (\%) & Without SPMs, N (\%) & With SPMs, N (\%) & $P$ value \\
\hline Age at diagnosis & & & & $<0.001$ \\
\hline$<35$ & $5,729(16.7)$ & $5,572(17.3)$ & $157(7.3)$ & \\
\hline $35 \sim 49$ & $14,663(42.8)$ & $13,943(43.4)$ & 720 (33.4) & \\
\hline$\geq 65$ & 4,532 (13.2) & $4,059(12.6)$ & $473(22.0)$ & \\
\hline Race & & & & 0.001 \\
\hline White & $26,216(76.4)$ & $24,603(76.5)$ & $1,613(74.9)$ & \\
\hline Black & 4,730 (13.8) & 4,376 (13.6) & 354 (16.4) & \\
\hline Squamous cell & $23,902(69.7)$ & $22,395(69.7)$ & $1,507(70.0)$ & \\
\hline Adenocarcinoma & 7,279 (21.2) & $6,809(21.2)$ & $470(21.8)$ & \\
\hline Others & $3,114(9.1)$ & $2,937(9.1)$ & $177(8.2)$ & \\
\hline SEER historic stage & & & & $<0.001$ \\
\hline Distant & $3,582(10.4)$ & $3,468(10.8)$ & $114(5.3)$ & \\
\hline Regional & $12,818(37.4)$ & $11,929(37.1)$ & 889 (41.3) & \\
\hline Localized & $17,895(52.2)$ & $16,744(52.1)$ & $1,151(53.4)$ & \\
\hline Surgery & & & & 0.387 \\
\hline
\end{tabular}

SPMs, second primary malignancies.

CC diagnosis was 47.6 years in the full cohort and was 53.1 years among those who developed SPMs. The mean age at the second cancer diagnosis was 58.3 years. The demographic and clinicopathological characteristics of these patients are shown in Table 1 . Overall, $60.7 \%$ of CC patients without SPM were diagnosed before age 50 , while $59.3 \%$ of the patients with SPMs were aged $>50$ years. Besides, racially black $(13.8 \%)$ patients, patients with local or regional disease $(89.6 \%)$, and patients treated with radiotherapy $(24.7 \%)$ seemed to be vulnerable for a subsequent malignancy $(\mathrm{P}<0.001)$. However, no statistically significant associations were found in the comparisons of primary sites, histological types, or surgical treatments. Patients with SPMs experienced better OS within 6 years after initial diagnosis than those without $(\mathrm{P}<0.001$, Figure 2). The RMST of those with and without SPMs were 129.0 and 144.6 months, respectively $(\mathrm{P}<0.001$, Figure S2).

When the major sites of SPMs were compared, the lung, breast, female urogenital system (including the bladder, kidney, vagina, corpus uteri, ovary, and cervix uteri) were found to be the most common, accounting for $21.0 \%$, $18.8 \%$, and $12.0 \%$, respectively (Table 2). Moreover, we observed that SPMs in the lung were more common in 
elderly patients (chi-square test, $\mathrm{P}<0.001$ ). Besides, the median interval between the diagnosis of initial CC and the SPMs was 100 months for lung and bronchus cancer, and 62 months for female breast cancer.

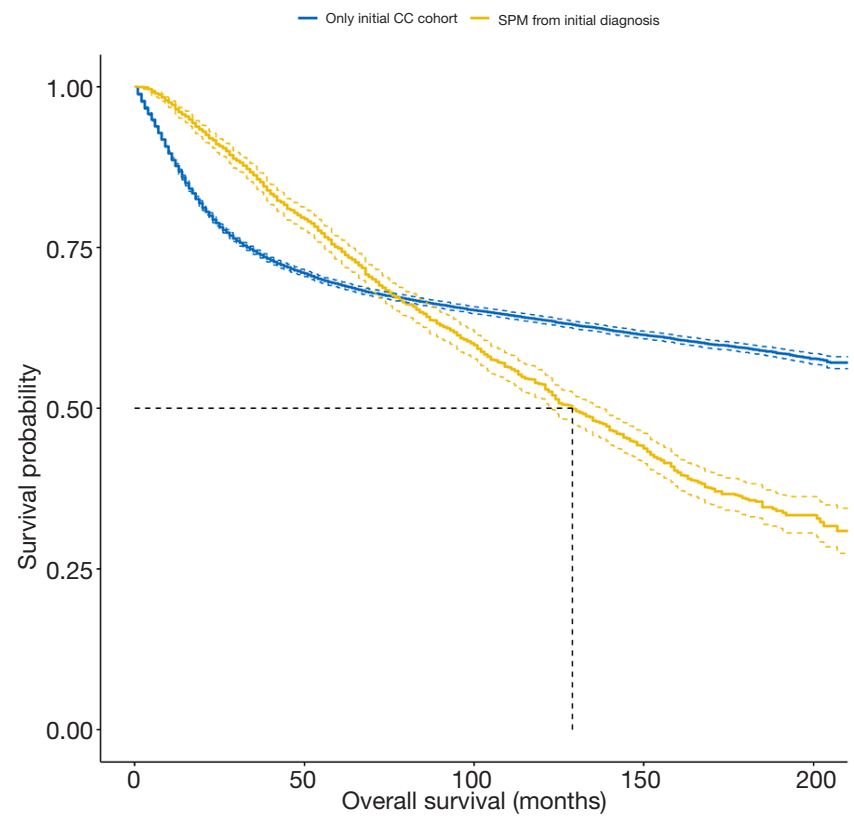

Figure $2 \mathrm{~K}-\mathrm{M}$ plot of overall survival (OS) for initial cervical cancer (CC) patients and for CC patients with second primary malignancies (SPM) from their initial diagnosis.

\section{Risk factors for developing SPMs}

Variables with $\mathrm{P}$ value $<0.05$ in Table 1 were further analyzed in the multivariable competing-risk analysis, and backward stepwise BIC selection was used to determine the most significant predictors. Four variables, including age, race, radiation treatment, and SEER historical stage, were retained in the final optimal model. Age $\geq 65$ (sdHR: 4.55; 95\% CI: 3.78-5.46; $\mathrm{P}<0.0001$ ), black race (sdHR: 1.42 ; 95\% CI: $1.19-1.70 ; \mathrm{P}<0.0001$ ), radiation treatment (sdHR: 1.25; 95\% CI: $1.14-1.38 ; \mathrm{P}<0.0001)$ and localized SEER historical stage (sdHR: 2.76; 95\% CI: 2.27-3.35; P<0.0001) were identified to be independently associated with a higher risk of SPMs (Table 3). The 3-, 5-, and 10-year cumulative incidences of the development of SPMs in CC survivors were also estimated (Figure 3).

\section{Nomogram for predictive cumulative incidence}

Based on the predictors mentioned above, a competingrisk nomogram was generated to estimate the 3-, 5-, and 10-year cumulative incidences of SPMs after the diagnosis of initial CC (Figure 4). Age emerged as the strongest predictor, followed by the SEER stage, race, and radiotherapy. Figure 4 demonstrates that the scoring with the nomogram effectively discriminated the risk of SPMs. Patients in the development group were stratified into three groups according to the predicted 10 -year risk

Table 2 Top cancer sites of SPMs after diagnosis of CC

\begin{tabular}{|c|c|c|c|c|}
\hline Site & N (\%) & \multicolumn{3}{|c|}{ Time to develop SPMs } \\
\hline Overall & 2,154 & 100 & 57 & 147 \\
\hline Lung and bronchus & $452(21.0)$ & 52 & 26 & 91.25 \\
\hline Female breast & 406 (18.9) & 62 & 25.5 & 100.25 \\
\hline $\begin{array}{l}\text { Female genital system } \\
\text { cancers except cervix } \\
\text { uteri cancer }\end{array}$ & $87(4.0)$ & 34 & 19 & 82.5 \\
\hline Cervix Uteri & $85(3.9)$ & 55 & 23 & 98 \\
\hline Thyroid & $85(3.9)$ & 44 & 12 & 93 \\
\hline
\end{tabular}

${ }^{*}$, urinary system cancers including cancers of bladder and kidney; ${ }^{* *}$, female genital system cancers except cervix uteri cancer including cancers of vagina, corpus uteri and ovary; ${ }^{* \star *}$, others including 68 kinds of different cancer sites and that the occurrence of these sites ranged from $2.3 \%$ to $0.4 \%$. SPMs, second primary malignancies; CC, cervical cancer. 
Table 3 Multivariable competing risk analysis for SPMs

\begin{tabular}{|c|c|c|c|}
\hline Variable & sdHR & $95 \% \mathrm{Cl}$ & $\mathrm{P}$ value \\
\hline$<35$ & Reference & & \\
\hline $35 \sim 49$ & 1.9 & $1.60-2.26$ & $<0.0001$ \\
\hline $50 \sim 64$ & 3.63 & $3.05-4.31$ & $<0.0001$ \\
\hline \multicolumn{4}{|l|}{ Race } \\
\hline Other & Reference & & \\
\hline Black & 1.42 & $1.19-1.70$ & $<0.0001$ \\
\hline White & 1.22 & $1.04-1.41$ & 0.0116 \\
\hline Regional & 2.32 & $1.91-2.82$ & $<0.0001$ \\
\hline Localized & 2.76 & $2.27-3.35$ & $<0.0001$ \\
\hline \multicolumn{4}{|l|}{ Radiation } \\
\hline No radiation & Reference & & \\
\hline Radiation & 1.25 & $1.14-1.38$ & $<0.0001$ \\
\hline
\end{tabular}

SPMs, second primary malignancies.

using the nomogram: the high risk ( $>75$ th percentile of the group, points: 164.1$)$, medium risk (25th-75th percentile of the group, points: 122.2$)$ and low-risk $(<25$ th percentile of the group, points: 109.3). The weight of each factor involved in the nomogram and the score of each patient are summarized in Figure S3. By calculating the total score of these clinical features, the risk of SPMs across time can be easily determined. The calibration curve showed good agreement between the observed incidences and predictions, with the data points close to the $45^{\circ}$ diagonal line (Figure 5). Moreover, for predicting the 5-and 10-year probabilities of developing SPMs after the diagnosis of CC, the DCA demonstrated that the clinical net benefit of the nomogram was larger than that in hypothetical alldeveloping or non-developing scenarios (Figure 6) for a wide range of thresholds (3.5\% to $14.0 \%$ ).

\section{Discussion}

This current study used the SEER database, which was considered the gold standard for cancer registries in the US and globally (22), to profile the characteristics of SPMs in CC survivors. As noted, $6.3 \%$ of those patients developed subsequent cancer within a median follow-up time of 7.1 years, and those with SPMs tended to be older, black in race, with localized disease, and underwent radiotherapy treatment. By analyzing the CC patients' survival outcomes in different periods, we found that 6.4 years after the initial CC diagnosis, the prognosis in patients with SPMs was significantly worse than those without. This indicated that metachronous SPMs showed a significant late survival disadvantage due to both increased physical and psychological burden (23). However, current followup guidelines mainly focus on the recurrence or metastasis of $\mathrm{CC}$, and insufficient attention has been paid to $\mathrm{CC}$ survivors.

Considering that approximately 1 in 16 CC survivors was observed to develop SPMs, intensive surveillance for SPMs in all CC survivors seems impractical. Therefore, a prediction tool to identify high-risk CC patients who may develop SPMs is an urgent need. In our present study, a competing-risk model-based nomogram was proposed as a convenient tool for clinicians to determine the high-risk CC survivors. Age, race, SEER historical stage, and radiotherapy were the potential risk factors for the development of SPMs. Ironically, the longer survival after the initial CC diagnosis 

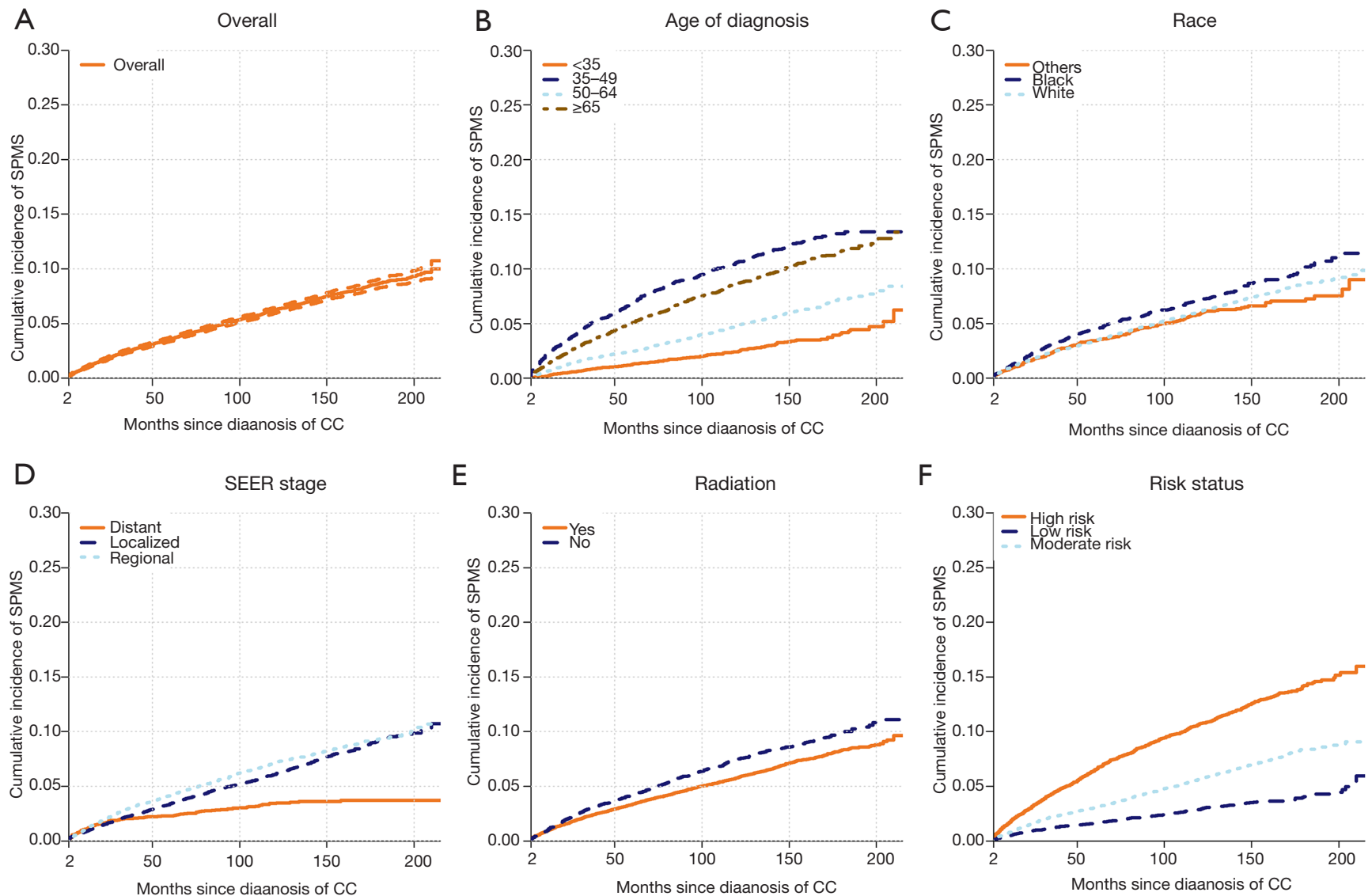

Figure 3 Cumulative incidence function (CIF) of cervical cancer (CC) patients developing a second primary malignancy (SPM) by subgroups, taking death as a competing event. (A) Overall CIF; (B) age at initial diagnosis; (C) race; (D) SEER stage; (E) radiation; (F) risk status.

Points

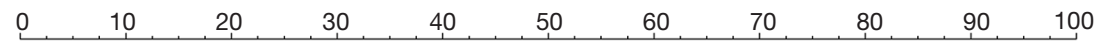

Age at diagnosis

Race

SEER stage

Radiation

Total points

3-year SPM Prob.

5-year SPM Prob.

10-year SPM Prob.

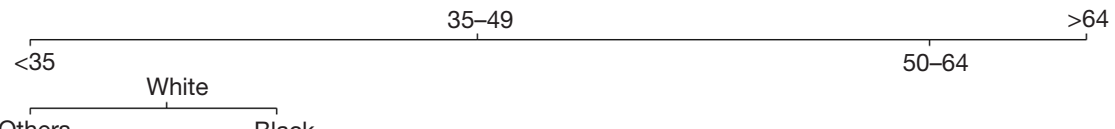

\begin{tabular}{llll} 
& White & & \\
Others & & Black & Regional \\
\cline { 3 - 3 } & Yes & & Localized \\
& &
\end{tabular}

No

\begin{tabular}{|c|c|c|c|c|c|c|c|c|c|c|c|c|}
\hline 0 & 20 & 40 & 60 & 80 & 100 & 120 & 140 & 160 & 180 & 200 & 220 & 240 \\
\hline & & & & 0.01 & & 0.02 & 0.03 & 0.04 & 0.05 & 0.060 .07 & 0.080 .09 & \\
\hline
\end{tabular}

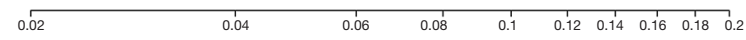

Figure 4 Nomogram for predicting the 3-, 5-, and 10-year probabilities of developing a second primary malignancy (SPM) among cervical cancer (CC) patients. 


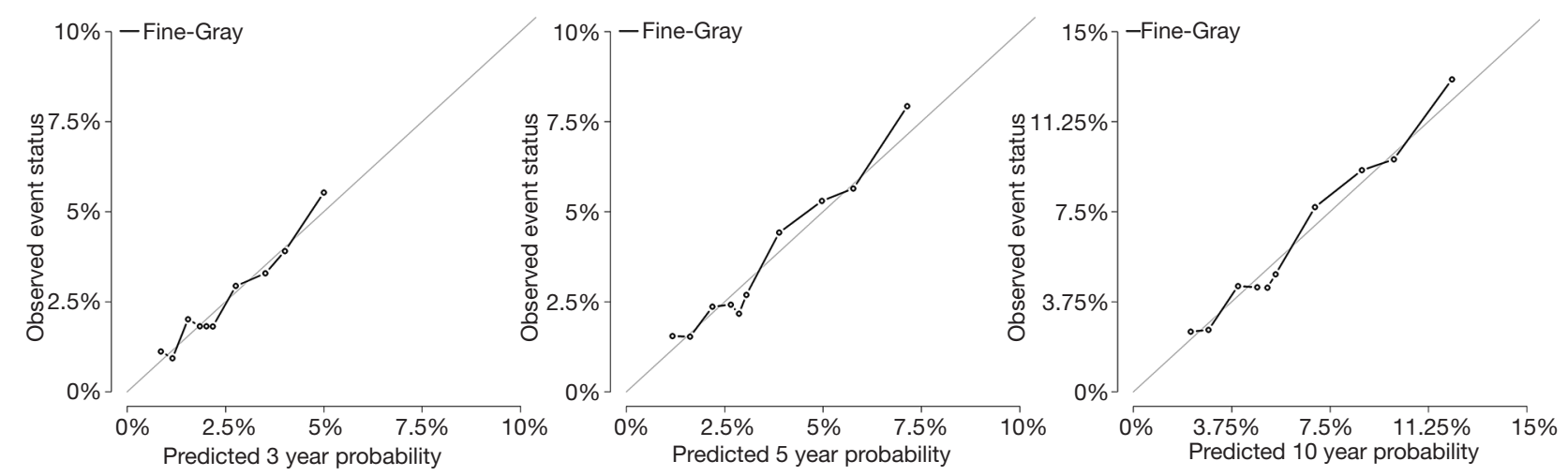

Figure 5 Calibration plot for the 3-, 5-, and 10-year prediction. (A) The predicted 3-year probability. (B) The predicted 5-year probability. (C) The predicted 10-year probability. X-axis: predicted the 3-, 5-, and 10-year probabilities of developing a second primary malignancy based on the multivariable competing risk model. Y-axis: observed cumulative probability.

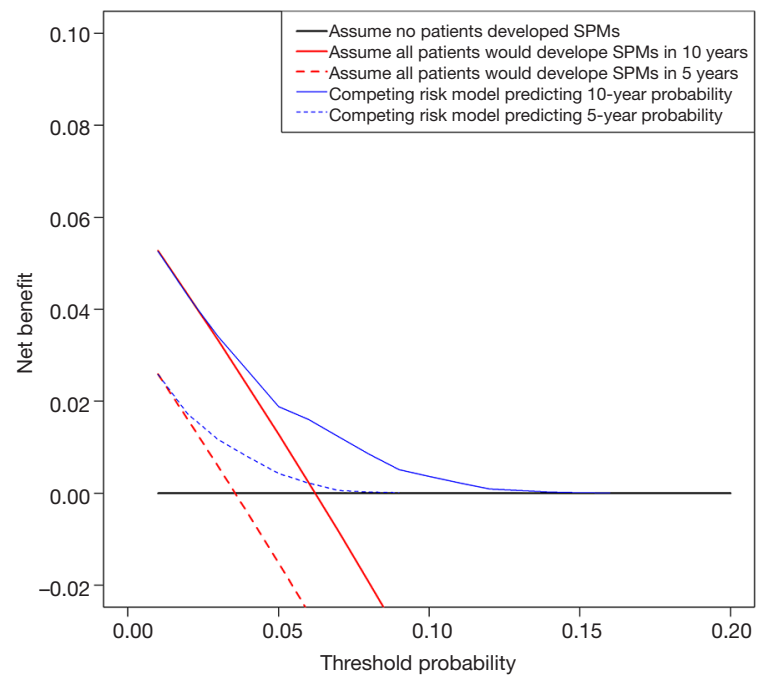

Figure 6 DCA for the competing-risk nomogram. The y-axis represents the net benefit. The two blue lines represent the 5-, and 10-year probabilities competing-risk nomogram. The two red lines represent the hypothesis that all patients had SPMs in 5 and 10 years. The solid black line represents the hypothesis that no patients had an SPM. The X-axis represents the threshold probability. The decision curve showed that if the threshold probability was between $3.5 \%$ and $14 \%$, then using the competingrisk nomogram to predict the probability of developing SPMs added more benefit than treating either all or no patients would have an SPM.

led to an extended risk period and increased prevalence of SPMs, and several possible explanations were proposed. For example, older patients, especially those $>50$ years old, had almost twice the risk of SPMs. Considering the critical role of estrogen and its receptor in the process of tumor development, this phenomenon could be partly explained by the fluctuation of estrogen secretion during aging, especially during the perimenopausal period (24). Estrogenrelated cancer development has also been documented in lung cancer and breast cancer $(25,26)$. Moreover, due to the weakening of the immune surveillance after age 50 , another potential mechanism is the increased risk of human papillomavirus-related malignancies (27).

The cumulative risk for SPMs was also higher in patients with localized disease. SEER stage, also known as the historic stage or summary stage, is the most basic way of categorizing how far a tumor has spread from its point of origin (28). Our results showed that the risk of SPM was higher among distant $\mathrm{CC}$ survivors than those with localized CC. Among the CC patients with distant metastasis, the competing effect of death led to a relatively lower incidence of SPM in comparison to those with localized CC.

Our study also demonstrated that radiotherapy increased the risk of SPM in CC patients. The damaging effect of $\mathrm{X}$-ray beams towards genetic material in normal cells, and the unexpected sequela of radiotherapy, including secondary tumorigenesis, have been widely proposed by previous researchers (29-31). Moreover, the risk of SPMs was significantly associated with the doses and therapeutic regimen of radiotherapy. One study observed a highly significant linear dose-response correlation for the risk of secondary stomach cancer at distal sites that were closest to the radiotherapy fields and received the highest doses (32). The emerging use of intensity-modulated radiotherapy 
(IMRT) also accounts for this phenomenon since a larger volume of normal tissue is exposed to lower radiation doses, which is a result of the increased number of monitor units and radiation leakage in IMRT (33). Besides, the risk of SPMs caused by radiotherapy varies in different sites. Chen et al. found that occurrence of SPMs increased among the moderately and heavily irradiated sites, including the bladder, vagina, corpus uteri, and kidney (11). Taken together, radiotherapy and aging are both risk factors for SPMs, and the interaction between them still needs further exploration, as women who have acquired radiation therapy may undergo premature menopause $(25,34)$. Therefore, physicians should weigh the benefits and potential threats more cautiously when prescribing radiotherapy to CC patients.

Current surveillance strategies have been generally aimed at screening $\mathrm{CC}$ for the recurrence or metastasis, especially the adjacent abdominal and pelvic organs, within five years postoperatively (34). Our findings suggest SPMs screenings should also be considered in the follow-up, and intensive follow-up strategies should be applied to highrisk SPM survivors (those $>24$ points according to our proposed nomogram). Close screening of gynecological tumors near the abdominal cavity should be given to those treated with radiotherapy every 3 to 6 months in the first 2 years. The screening of lung and breast cancer using sensitive biomarkers like CEA and CA199 and low-dose CT or breast ultrasonography should be done every 6 to 12 months in the next 3 to 5 years so as to detect these diseases as early as possible before the emergence of visible imaging evidence.

Although our study tried to overcome the shortcomings of previous studies, it still had inevitable limitations. First, acknowledged risk factors, such as HPV infection status (35), smoking status (36), family history, comorbidities, and detailed treatment regimens were not provided in the SEER database, thus limiting our ability to assess other clinical treatment modalities. Second, we failed to evaluate the detailed technique and dose of radiotherapy (37). Third, although we restricted our analyses to patients who were followed for more than 2 months after their initial CC to exclude synchronous cancers, it was possible that metastasis and recurrence of CC occurred later on in the study. Nevertheless, the definition of SPMs in the SEER database is strictly defined, and the SEER database is one of the best databases in cancer registration systems worldwide. Fourth, considering that treatment regimens during 1998-2010 might have shifted over time and may confound results, we decided to remove such patients. Further exploration in this field is needed to clarify the possible risk factors of SPMs and to provide an optimal long-term monitoring strategy for highrisk populations.

\section{Conclusions}

In conclusion, we developed a prediction model for SPMs risk based on clinical and demographic risk factors using data from a large population-based cohort. Our data confirmed that older age at diagnosis, black race, localized SEER stage, and radiation treatment were associated with an increased risk of SPMs after CC. The nomogram could help clinicians to determine the effectiveness of treatment for a given patient. Precautions on targeted surveillance and screening strategies for SPMs in high-risk CC survivors are warranted.

\section{Acknowledgments}

We extend our thanks to Dr. Guoshu Bi for all his valuable advice.

Funding: None.

\section{Footnote}

Reporting Checklist: The authors have completed the TRIPOD reporting checklist. Available at http://dx.doi. org/10.21037/atm-20-2003

Conflicts of Interest: All authors have completed the ICMJE uniform disclosure form (available at http://dx.doi. org/10.21037/atm-20-2003). The authors have no conflicts of interest to declare.

Ethical Statement: The authors are accountable for all aspects of the work in ensuring that questions related to the accuracy or integrity of any part of the work are appropriately investigated and resolved. The study was conducted in accordance with the Declaration of Helsinki (as revised in 2013).

Open Access Statement: This is an Open Access article distributed in accordance with the Creative Commons Attribution-NonCommercial-NoDerivs 4.0 International License (CC BY-NC-ND 4.0), which permits the noncommercial replication and distribution of the article with 
the strict proviso that no changes or edits are made and the original work is properly cited (including links to both the formal publication through the relevant DOI and the license). See: https://creativecommons.org/licenses/by-nc-nd/4.0/.

\section{References}

1. Organization WH. Early diagnosis and screening Cervical cancer. 2018. Available online: https://www. who.int/cancer/prevention/diagnosis-screening/cervicalcancer/en/

2. Cancer IAfRo. Cervical uteri - Estimated incidence, mortality and prevalence worldwide in 2018. 2018. Available online: https://gco.iarc.fr/today/fact-sheetscancers.

3. Institute NC. Five-Year Survival Rates of Cervical Cancer. Physician Data Query system. 2002. Available online: https://training.seer.cancer.gov/cervical-uterine/cervix/ intro/survival.html

4. Eskander RN, Tewari KS. Immunotherapy: an evolving paradigm in the treatment of advanced cervical cancer. Clin Ther 2015;37:20-38.

5. Murphy CC, Gerber DE, Pruitt SL. Prevalence of Prior Cancer Among Persons Newly Diagnosed With Cancer: An Initial Report From the Surveillance, Epidemiology, and End Results Program. JAMA Oncol 2018;4:832-6.

6. Le Borgne G, Mercier M, Woronoff AS, et al. Quality of life in long-term cervical cancer survivors: a populationbased study. Gynecol Oncol 2013;129:222-8.

7. Hildebrand P, Humke J, Oevermann E, et al. Influence of second or multiple tumours on the prognosis of patients with colorectal cancer. Acta Chir Iugosl 2012;59:31-8.

8. Howell R. Second Primary Cancers and Cardiovascular Disease after Radiation Therapy. NCRP Report No. 170. Med Phys 2012;39:7729-31.

9. Lim MC, Won YJ, Lim J, et al. Second Primary Cancer after Diagnosis and Treatment of Cervical Cancer. Cancer Res Treat 2016;48:641-9.

10. Donin N, Filson C, Drakaki A, et al. Risk of second primary malignancies among cancer survivors in the United States, 1992 through 2008. Cancer 2016;122:3075-86.

11. Chen CY, Lai CH, Lee KD, et al. Risk of second primary malignancies in women with cervical cancer: a populationbased study in Taiwan over a 30-year period. Gynecol Oncol 2012;127:625-30.

12. Teng CJ, Huon LK, Hu YW, et al. Secondary Primary Malignancy Risk in Patients With Cervical Cancer in
Taiwan: A Nationwide Population-Based Study. Medicine (Baltimore) 2015;94:e1803.

13. Austin PC, Lee DS, Fine JP. Introduction to the Analysis of Survival Data in the Presence of Competing Risks. Circulation 2016;133:601-9.

14. Collins GS, Reitsma JB, Altman DG, et al. Transparent reporting of a multivariable prediction model for individual prognosis or diagnosis (TRIPOD): the TRIPOD Statement. BMC Med 2015;13:1.

15. Institute NC. About the SEER Registries. 2020. Available online: https://seer.cancer.gov/registries/

16. Curtis RE, Freedman DM, Ron E, et al. New Malignancies Among Cancer Survivors: SEER Cancer Registries, 19732000. No. 05-5302. Bethesda, MD: National Cancer Institute, 2006.

17. Scrucca L, Santucci A, Aversa F. Regression modeling of competing risk using $\mathrm{R}$ : an in depth guide for clinicians. Bone Marrow Transplant 2010;45:1388-95.

18. Kuk D, Varadhan R. Model selection in competing risks regression. Stat Med 2013;32:3077-88.

19. Royston P, Parmar MK. Restricted mean survival time: an alternative to the hazard ratio for the design and analysis of randomized trials with a time-to-event outcome. BMC Med Res Methodol 2013;13:152.

20. Iasonos A, Schrag D, Raj GV, et al. How to build and interpret a nomogram for cancer prognosis. J Clin Oncol 2008;26:1364-70.

21. Vickers AJ, Van Calster B, Steyerberg EW. Net benefit approaches to the evaluation of prediction models, molecular markers, and diagnostic tests. BMJ 2016;352:i6.

22. Duggan MA, Anderson WF, Altekruse S, et al. The Surveillance, Epidemiology, and End Results (SEER) Program and Pathology: Toward Strengthening the Critical Relationship. Am J Surg Pathol 2016;40:e94-e102.

23. Thong MS, Mols F, Verhoeven RH, et al. Multiple primary cancer survivors have poorer health status and well-being than single primary cancer survivors: a study from the population-based PROFILES registry. Psychooncology 2013;22:1834-42.

24. Nelson HD. Menopause. Lancet 2008;371:760-70.

25. Einstein MH, Levine NF, Nevadunsky NS. Menopause and Cancers. Endocrinol Metab Clin North Am 2015;44:603-17.

26. Yager JD, Davidson NE. Estrogen carcinogenesis in breast cancer. N Engl J Med 2006;354:270-82.

27. Barros MR Jr, de Melo CML, Barros M, et al. Activities of stromal and immune cells in HPV-related cancers. J Exp Clin Cancer Res 2018;37:137. 
28. Institute NC. Summary Stage 2018 General Coding Instructions. 2019. Available online: https://seer.cancer. gov/tools/ssm/

29. Arnold M, Liu L, Kenter GG, et al. Second primary cancers in survivors of cervical cancer in The Netherlands: Implications for prevention and surveillance. Radiother Oncol 2014;111:374-81.

30. Papatla K, Houck KL, Hernandez E, et al. Second primary uterine malignancies after radiation therapy for cervical cancer. Arch Gynecol Obstet 2019;300:389-94.

31. Ota T, Takeshima N, Tabata T, et al. Treatment of squamous cell carcinoma of the uterine cervix with radiation therapy alone: long-term survival, late complications, and incidence of second cancers. Br J Cancer 2007;97:1058-62.

32. Kleinerman RA, Smith SA, Holowaty E, et al. Radiation dose and subsequent risk for stomach cancer in long-term survivors of cervical cancer. Int J Radiat Oncol Biol Phys

Cite this article as: Li R, Zhang Y, Ma B, Tan K, Lynn HS, $\mathrm{Wu}$ Z. Survival analysis of second primary malignancies after cervical cancer using a competing risk model: implications for prevention and surveillance. Ann Transl Med 2021;9(3):239. doi: 10.21037/atm-20-2003
2013;86:922-9.

33. Hall EJ. Intensity-modulated radiation therapy, protons, and the risk of second cancers. Int J Radiat Oncol Biol Phys 2006;65:1-7.

34. Koh WJ, Greer BE, Abu-Rustum NR, et al. Cervical cancer. J Natl Compr Canc Netw 2013;11:320-43.

35. Papatla K, Halpern MT, Hernandez E, et al. Second primary anal and oropharyngeal cancers in cervical cancer survivors. Am J Obstet Gynecol 2019;221:478 e1-e6.

36. Underwood JM, Rim SH, Fairley TL, et al. Cervical cancer survivors at increased risk of subsequent tobaccorelated malignancies, United States 1992-2008. Cancer Causes Control 2012;23:1009-16.

37. Lee B, Ahn SH, Kim H, et al. Secondary cancer-incidence risk estimates for external radiotherapy and high-dose-rate brachytherapy in cervical cancer: phantom study. J Appl Clin Med Phys 2016;17:124-32. 


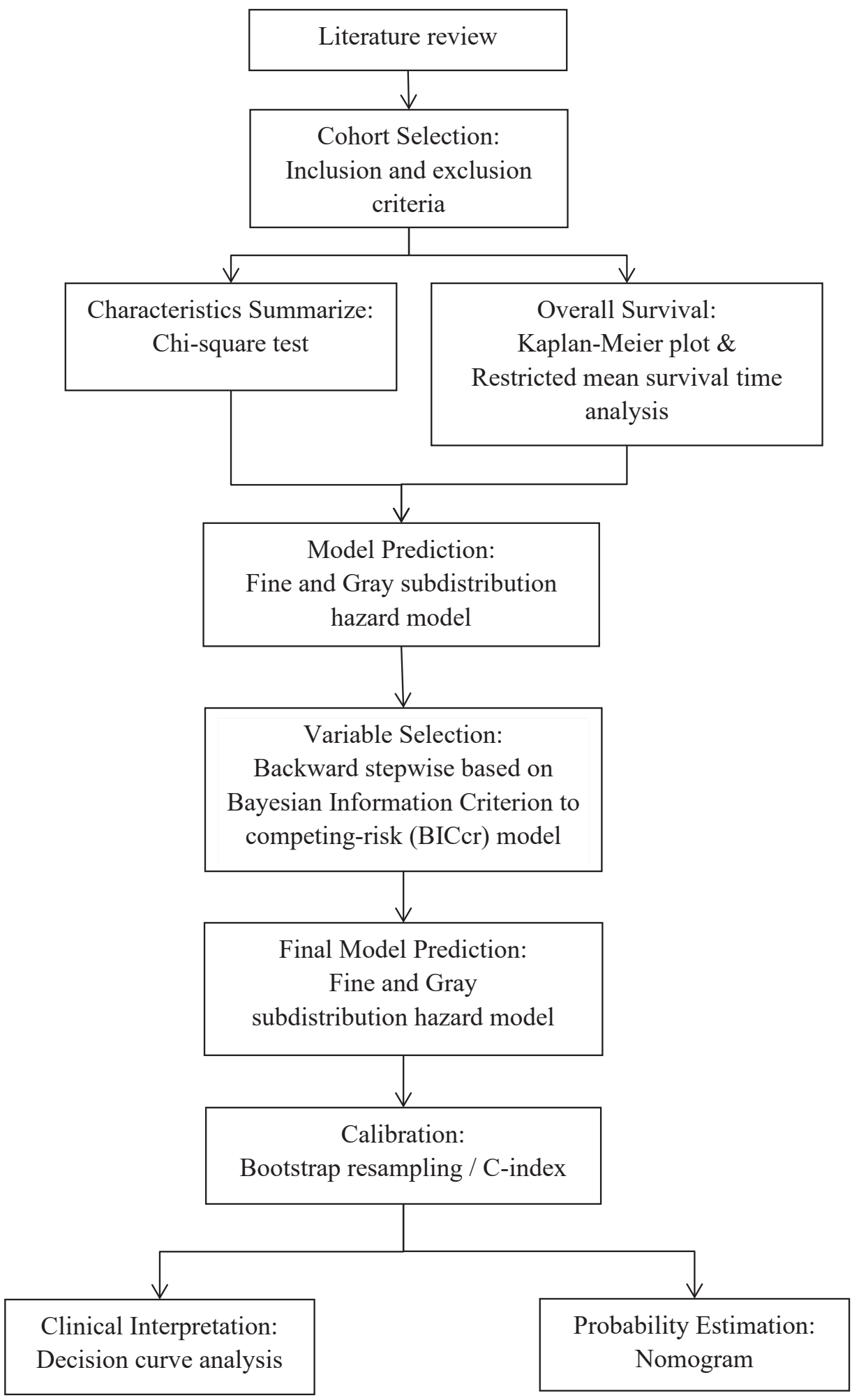

Figure S1 Overview of the case study design. 

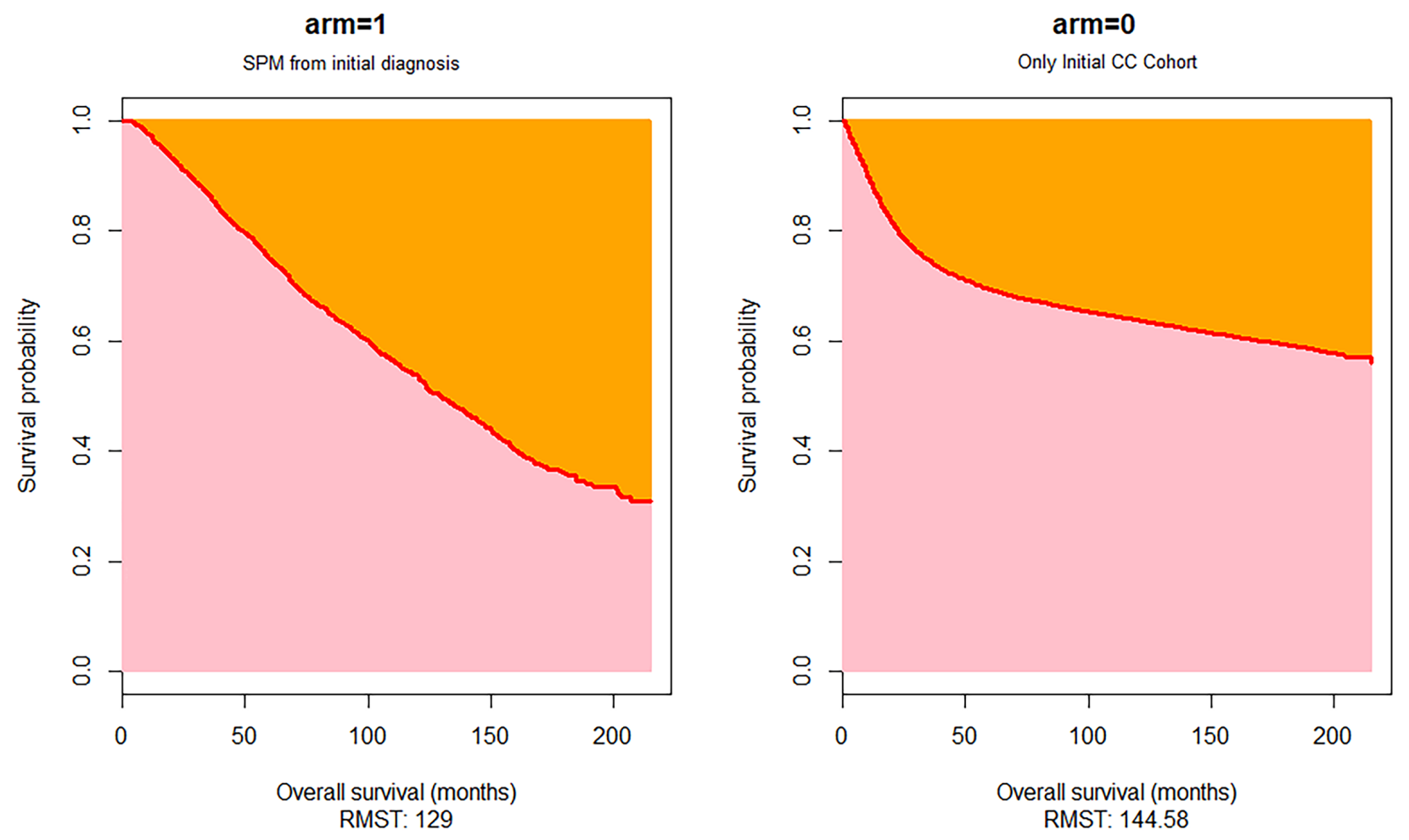

Figure S2 Restricted mean survival time (RMST) of CC patients with and without SPM.

\begin{tabular}{|l|l|l|l|}
\hline cut-off & low risk (1st quantile) & medium risk (median) & high risk (3rd quantile) \\
\hline points & 109.3 & 122.2 & 164.1 \\
\hline
\end{tabular}

Figure S3 The weight of each factor involved in the nomogram and the score of each patient. 